\title{
Vascular Endothelial Growth Factor: Not Only for Vessels Anymore
}

\author{
A review of: Compernolle V, Brusselmans K, Acker T et al. 2002 Loss of HIF-2a and inhibition of VEGF impair fetal \\ lung maturation, whereas treatment with VEGF prevents fatal respiratory distress in premature mice. Nat Med \\ 8:702-710 (Also see accompanying editorial: Rabinovitch M, Bland R 2002 Novel notions on newborn lung disease.
} Nat Med 8:664-666)

$\mathrm{V}$ ASCULAR ENDOTHELIAL GROWTH factor (VEGF) is a potent endothelial cell mitogen and pro-angiogenic agent that regulates vascular growth in the systemic and pulmonary circulations, as well as in tumors. VEGF is an especially critical mediator of normal lung vascularization during development and coordinates essential interactions between airway and blood vessels that are required for the establishment of normal lung architecture. During early fetal life, VEGF is strongly expressed at tips of branching airways, where VEGF stimulates vascular growth through activation of specific receptors located on endothelial cells. Clinically, VEGF levels in tracheal fluid samples and lung tissue from infants with bronchopulmonary dysplasia (BPD) are markedly reduced, implicating a role in its pathogenesis $(1,2)$. Pharmacologic inhibition of VEGF receptors reduces alveolarization in infant rats, suggesting that impaired VEGF signaling can contribute to failed lung vascular and alveolar growth in BPD (3). More recent studies have challenged the current paradigm that the exclusive role of VEGF during lung development is through its regulation of endothelial cell growth and function. Dr. Acarregui's laboratory has previously shown that airway epithelial cells can express VEGF receptors and that VEGF stimulates lung epithelial cell proliferation in vitro (4). However, the role of endogenous VEGF on airway growth and development in vivo has been uncertain.

This current paper, which represents extensive work from several collaborating laboratories under the direction of Dr. Carmeliet, convincingly demonstrates that VEGF is also a lung epithelial cell growth and differentiation factor and maturational stimulus for surfactant production (5). These conclusions are drawn form an extensive series of experiments involving mice that are genetically deficient for hypoxia inducible factor $-2 \mathrm{a}$ (HIF-2a). HIF-2a -/- mice have deficient lung surfactant and die from cardiorespiratory failure shortly after birth. Although histology suggests normal lung structure during

\section{Steven Abman}

the early stages of lung development, lungs from HIF-2a $-/-$ mice have interstitial thickening and immature-appearing type II epithelial cells in the saccular phase. These cells are characterized by the persistence of large glycogen stores, which are characteristic of immature epithelium. HIF-2a -/- mice also have reduced lung phospholipid content, decreased surfactant protein expression and fewer type II cells, without apparent affects on neuroepithelial or Clara cells.

Since VEGF is a known downstream target of HIF-2a, lung VEGF expression was measured from late fetal mice. In comparison with wild type controls, lung VEGF content is markedly reduced in HIF-2a -/- mice. Additional studies show that mice deficient in some VEGF isoforms $\left(\mathrm{VEGF}_{164}\right.$ and $\mathrm{VEGF}_{188}$ ) die from neonatal respiratory distress syndrome (RDS), as found in the HIF-2a $-/-$ mice. To determine the effects of exogenous VEGF treatment on RDS, premature mice were treated with intratracheal (postnatal) or intra-amniotic (prenatal) injections of VEGF, and were then examined for visible signs of distress (by modified APGAR scores) and survival. Although control mice died within 10 hours after delivery, VEGF treatment improved APGAR scores and prolonged survival within the first 20 hours of life.

Overall, these findings suggest that: HIF-2a plays an important role in type II cell differentiation and function during late gestation; HIF-2a deficiency causes neonatal RDS and downregulates impaired VEGF expression and activity; and that VEGF treatment improves cardiorespiratory function and prolongs survival of premature mice with RDS. Data on the effects of VEGF treatment of HIF-2a $-/-$ mice is noticeably absent from this paper, which is a critical link in this hypothesis. Another limitation is the lack of physiologic studies that actually define mechanisms underlying cyanosis, respiratory distress and death in these mouse models. Al- though neonatal RDS in the HIF-2a and VEGF deficient mice is likely due to surfactant deficiency, it is possible that altered cardiac or lung vascular function may further contribute to the failure of cardiopulmonary transition in these models.

Thus, this study provides novel and important new findings regarding basic mechanisms regarding the roles of HIF-2a and VEGF during late lung development and their effects on type II cell maturation. These findings may lead to novel strategies for the future treatment of premature newborns with severe RDS.

1. Lassus P, Turanlahti M, Heikkila P, Anderson LC, Nupponen I, Sarnesto A, Andersson S 2001 Pulmonary VEGF and Flt-1 in fetuses, acute and chronic lung disease, and in PPHN. Am J Respir Crit Care Med 164:1981-1987

2. Bhatt AJ, Pryhuber GS, Huyck H, Watkins RH, Metlay LA, Maniscalco WM 2001 Disrupted pulmonary vasculature and decreased vascular endothelial growth factor, Flt-1 and Tie-2 in human infants dying with BPD. Am J Respir Crit Care Med 164:1971-1980

3. Jakkula M, Le Cras TD, Gebb S, Hirth KP, Tuder RM, Voelkel NF, Abman SH 2000 Inhibition of angiogenesis decreases alveolarization in the developing rat lung. Am J Physiol Lung Cell Mol Physiol 279:L600-607

4. Brown KR, England KM, Goss KL, Snyder JM, Acarregui MJ 2001 VEGF induces airway epithelial cell proliferation in human lung in vitro. Am J Physiol Lung Cell Mol Physiol 281:L1001-1010

5. Compernolle V, Brusselmans K, Acker T, Hoet P, Tjwa M, Beck H, Plaisance S, Dor Y, Keshet E, Lupu F, Nemery B, Dewerchin M, Van Veldhoven P, Plate K, Moons L, Collen D, Carmeliet P 2002 Loss of HIF-2a and inhibition of VEGF impair fetal lung maturation, whereas treatment with VEGF prevents fatal respiratory distress in premature mice. Nat Med 8:702-710

Pulmonary Medicine, B395

The Children's Hospital

1056 E. Nineteenth Ave.

Denver, CO 80210

U.S.A.

steven.abman@uchsc.edu

DOI: 10.1203/01.PDR.0000047500.60130.90 